\title{
Moving plate in a nanofluid using Buongiorno model and Thermophysical properties of nanoliquids
}

\begin{abstract}
The steady two dimensional boundary layer flow of a nanofluid on a moving plate is studied numerically. We focus on silver $(\mathrm{Ag})$, copper $(\mathrm{Cu})$, alumina and titania nanoparticles in water based fluid. The partial differential equations are converted into nonlinear ordinary differential equations by using similarity transformations and have been solved numerically using shooting method. Ag-water fluid is found to be pronounced than the other nanoparticles. The dual solutions are found in a certain range of the pertinent parameters.
\end{abstract}

Keyword: Boundary layer flow; Nanofluid; Moving plate; Heat transfer; Dual solutions 